\title{
LA FILOSOFÍA DE LA ACCIÓN COMO BASE TEÓRICA DE LA INVESTIGACIÓN SOCIAL, EL CASO DE LAS MIGRACIONES ${ }^{1}$
}

\author{
JORGE GREGORIO POSADA RAMÍREZ \\ Programa de Filosofía, Universidad del Quindío, Colombia \\ gposada@uniquindio.edu.co
}

Manizales, 2010-02-08 (Rev. 2010-05-14).

\section{RESUMEN}

El siguiente artículo está dividido en tres partes. En la primera se describen las tesis fundamentales que configuran la renovada tradición filosófica de la filosofía de la acción; se muestran sus fundamentos filosóficos y sus diferencias con los sucesos naturales. En la segunda parte, y a partir de la noción que se ha propuesto de lo que son las acciones, se argumenta que es promisorio asumir a las acciones como el objeto de estudio de las ciencias sociales; en términos técnicos, se sostiene que las acciones son la ontología de la realidad social. En la tercera parte, a partir de la revisión de algunos informes acerca de la migración en la región cafetera, se muestra la posibilidad real de que los investigadores de las ciencias sociales se valgan de la filosofía de la acción como parte de sus presupuestos teóricos de investigación.

\section{PALABRAS CLAVE:}

Filosofía, ontología social, acciones, migraciones, causas naturales, razones como causas humanas.

\section{THE PHILOSOPHY OF ACTION AS A THEORETICAL BASE FOR SOCIAL RESEARCH, THE MIGRATION CASE}

\begin{abstract}
The following article is divided into three parts. In the first, the fundamental theses that give form to the renewed philosophical tradition to the philosophy of action are described; their philosophical base and differences with natural events are showed. In the second part, and starting from the proposed notion of what actions are, it is argued that it is promissory to assume actions as the object of study of social sciences; in technical language, it is maintained that the actions are the ontology of social reality. In the third part, beginning with the inquiry of some reports that deal with migration in the coffee region, it shows the real possibility that social science researchers may use philosophy of action as a part of their theoretical premises of research.
\end{abstract}

KEY WORDS: philosophy, social ontology, actions, migrations, natural causes, reasons as human causes.

Aristóteles en la Ética Nicomaquea distingue entre aquellos acontecimientos que tienen como causas disposiciones naturales y aquellos que se dan gracias a que 
el hombre es su principio generador. Con el propósito, entre otros, de establecer cuándo algunos acontecimientos merecen evaluación moral y cuándo son ajenos al juicio de loables o censurables, Aristóteles cualifica las acciones humanas como aquellas en donde las personas son su principio causal "como los hijos son de sus padres", como diría Aristóteles (Ética Nicomaquea, libro 3, capítulo 5). Esto, en contraposición a los fenómenos que ocurren con independencia de la asistencia relevante de los hombres, en este caso, Aristóteles habla de Dinamis, definiéndola como la capacidad o aptitud natural de los entes o de la materia a operar. Y en donde la evaluación moral no es aplicable.

Determinismo causal, en el caso de los sucesos naturales, actos de la volición en el caso de las acciones humanas, son las distinciones grosso modo descritas por Aristóteles y que hoy utilizan algunos filósofos para marcar la diferencia entre acciones y acontecimientos que no son acciones, e iniciar así sus estudios. Ambos conceptos: determinismo causal, actos de la volición, son la base para la clarificación filosófica de lo que son las acciones. Así, por ejemplo, el movimiento de las placas tectónicas es originado por ciertas leyes deterministas que operan en la materia, leyes que causan necesariamente el movimiento de estos bloques rígidos. De otro lado, el desplazamiento masivo y constante de un grupo de personas de su país de origen a otro lugar, no obedece a determinaciones causales de la materia, sino a las decisiones racionales de las personas, así éstas en muchos casos sean limitadas. O para contrastar con otro ejemplo, mientras las aves migran de norte a sur predeterminadas por patrones biológicos e instintivos, algunos colombianos migran de sur a norte, no constreñidos por factores biológicos o instintivos, sino por cierto tipo de razones que los llevan a actuar. En el caso de las placas tectónicas como en el de las aves, el principio de sus movimientos está en el determinismo causal, en el caso de la migración humana está en los actos de la volición.

No obstante, a pesar de las diferencias entre estos tipos de desplazamiento, los tres tienen algo en común: son originados y explicados por su causa. Así, el determinismo causal, y los actos de la volición, son ambos un tipo de relación causal. Lo que es o no es una acción, como espero empiece a verse, depende del tipo de relación causal que dé origen y explicación a los fenómenos. Ya podemos adelantar que el determinismo causal está del lado de los acontecimientos que no son acciones, y que los actos de la volición o actos de la voluntad, están del lado de lo que son las acciones.

Tanto el determinismo causal como los actos de la volición, son conceptos conectados con un concepto más básico: el de que todo tiene una causa. Un concepto es básico cuando está en el núcleo de nuestra imagen racional del mundo, cuando en él descansa en gran medida nuestra imagen coherente de las cosas, y cuando es justamente nuestra creencia en él, la que ha permitido nuestras formas exitosas de lidiar con el mundo. El concepto de causa es uno de ellos. Y es además el concepto básico en la filosofía de la acción, pues digámoslo de una vez, prima facie las acciones son acontecimientos que se dan en el mundo causados por los hombres.

En filosofía, la creencia de que todo tiene una causa se justifica en el principio de que de la nada sólo se sigue la nada, por eso, lo que es ha de haber sido por otra cosa. Y como todo lo que sucede en el mundo, si sucede es, entonces todo lo que sucede en el mundo ha de haber tenido una causa. Incluyendo, obviamente, el desplazamiento de las placas tectónicas, la migración de las aves, y el éxodo de algunos colombianos. Con un ejemplo recreemos este tecnicismo filosófico.

Tres días después de pagar mi cuenta de servicio eléctrico me doy cuenta de 
que en mi apartamento ningún electrodoméstico enciende. Llamo al portero y él me dice que en el edificio no hay problemas con la energía, que en todos los apartamentos, excepto en el mío, hay fluido eléctrico. Con desconcierto llamo a la empresa de energía y la operadora me dice que en el sector en el que vivo todo está funcionando bien, que lo mejor es que me cerciore de haber pagado la factura. A lo que respondo que ya lo hice, constaté que efectivamente he pagado la factura de energía de mi apartamento. Indiferente la mujer del teléfono me responde que no hay una razón de mi situación, que seguramente es un caso en el que las cosas dejan de funcionar, porque sí; se dan porque se dan, y ya. Turbado voy personalmente a la empresa, con el recibo de pago en mano, y el funcionario que me atiende dice que efectivamente puede verse mi registro de pago, y que el servicio de energía anda bien en mi sector, pero que no hay una causa, no hay un porqué que explique lo que pasa en mi apartamento. Pero que no sienta extrañeza ni me preocupe, que el mío es un típico caso en el que a pesar de haberse pagado la factura y de que todo está normal, el servicio eléctrico no funciona sin ninguna causa que lo explique. Simplemente deja de funcionar en razón de nada, se daña porque sí, y es todo lo que hay que decir.

Nuestra resistencia a creer que de hecho existen tales casos, de que los problemas aparecen y ya; que a pesar de que paguemos nuestros recibos de la energía, ésta puede ser suspendida porque sí, y ya, revela nuestra creencia de que el mundo se comporta causalmente. Todo lo que sucede se da porque necesariamente algo lo causa o provoca. Creemos que todo tiene una causa, que las cosas no aparecen así como así, sino que siempre algo las causa. Desde esta creencia se construye nuestra interpretación de la naturaleza y nuestras expectativas del modo como se comporta el mundo.

Como ya mencionamos, para los filósofos que asumen el problema de la acción existen dos tipos de comportamientos causales: unos que obedecen a relaciones deterministas y otros que dependen de actos de la volición. Respecto al determinismo causal, se dice que un acontecimiento es causalmente determinante de otro, si es imposible que se dé el acontecimiento que es su causa sin su correspondiente efecto. Así, decimos que el cigarrillo encendido determina causalmente la aparición del humo, porque siempre que se enciende un cigarrillo se sigue necesariamente la aparición del humo. En el determinismo causal, dado un fenómeno no existe la posibilidad de que se dé otro distinto a aquel con el que el primero siempre ha estado asociado. Básicamente, el determinismo causal muestra que los acontecimientos naturales siguen leyes, y que de ser descubiertas esas leyes el hombre puede explicar y predecir el comportamiento de las cosas. Las placas tectónicas, la migración de las aves son casos de este tipo de relación causal. En virtud de ciertas leyes las placas se desplazan como lo hacen configurando el relieve terrestre, en virtud de ciertas condiciones antecedentes las aves migran hacia zonas cálidas. Valga decir, si cambian esas condiciones antecedentes cambian sus efectos, en este caso el movimiento de las placas y la migración de las aves. Es posible que la migración de las aves se vea afectada, si por ejemplo, la aparición del invierno, que es su causa, registra comportamientos irregulares; tal irregularidad se verá en el desplazamiento de las aves.

La fórmula de que se valen los filósofos para representar el determinismo causal es: de darse A necesariamente se da B. Como podrá colegirse, los fenómenos del mundo que son acciones no obedecen al determinismo causal, pues en el comportamiento de los hombres de darse A no necesariamente se da B. No obstante, registran otro tipo de relación causal, la marcada por lo que Aristóteles señala como actos de la volición, y que en la filosofía de la acción se reconoce como: razones. Desde la distinción entre determinismo causal y actos de la 
volición, se asume a las razones como las causas de las acciones. En la literatura filosófica esto se reconoce como razones para actuar. $^{2}$

\section{Razones como causas de la acción}

Desde el punto de vista externo, nada distingue los acontecimientos naturales de las acciones. Tanto la migración de las aves como la migración humana, registran externamente rasgos comunes: un grupo de miembros de una especie trasladándose de su lugar de origen a otro. Desde nuestra percepción en tercera persona, es decir, como perceptores neutrales que excluyen sus experiencias propias cuando observan, las acciones y los acontecimientos naturales son idénticos. Desde la tercera persona, los movimientos involuntarios de un hombre que guiña su ojo por un tic nervioso, en nada se distinguen de los de un hombre que lo hace siguiendo sus deseos de coquetear. La distinción aparece sólo cuando se asume que el hombre que guiñe su ojo coqueteando lo hace movido por el deseo y por las creencias de que así conseguirá dar señales de agrado. Deseos y creencias en conjunción configuran lo que son las razones para la acción. Ellos, creencias y deseos, son las causas de las acciones humanas. Así, todo fenómeno que tenga como origen causal creencias y deseos es una acción, los que no tengan esta historia causal, son simplemente fenómenos naturales, o si se quiere biológicos. Como afirma Bertrand Russell, en su libro El análisis del Espíritu: si bien, no hay una línea evolutiva continua entre los protozoarios y los humanos, es claro que la migración de los animales se debe a condiciones que son distintas a la de los animales humanos, y esta nota singular de los humanos ha de ubicarse en su vida mental antes que la biológica. La filosofía de la acción en comunión con la filosofía de la mente, denota esta singularidad del comportamiento humano en las nociones de razones, las cuales, y volvámoslo a decir, conjugan los estados mentales del creer y del desear.

Para sintetizar, una acción es un fenómeno que se da en el mundo causado por razones; las razones son la conjugación de dos clases de estados mentales: el creer y el desear. En consecuencia, las acciones son los eventos que se dan en el mundo, causados por creencias y deseos. Así, la migración de los colombianos se distingue de la migración de las aves, en tanto la migración de los primeros es causada por creencias y deseos, mientras que la migración de los segundos se da por causas deterministas.

\section{Consecuencias para las ciencias sociales}

Declarar que las acciones son todos aquellos fenómenos causados por las razones, y que esta singularidad causal es la que distingue los comportamientos de la especie humana, de todas las demás, afirmar esto, implica que el objeto de estudio de las ciencias sociales son las acciones. Pues si las ciencias sociales estudian aquellos rasgos singulares de la especie humana, aquello que la hace diferente de todos los demás organismos, la noción de acción, y los conceptos que la definen, como son el de razones, creencias, deseos e intenciones recogen justamente esta singularidad. Si bien, un hombre que se arroja desde un peñasco se acelera a la misma velocidad que una piedra que cae a su lado, y eventualmente, puede destrozarse tal como la piedra, el estudio de la naturaleza humana, el estudio de las ciencias sociales, no ha de considerar al hombre como un cuerpo más de la naturaleza, o una partícula con una masa cuantificable. Las personas piensan, actúan, hablan, y las ciencias sociales en tanto estudian los rasgos singulares del hombre se refieren al pensamiento, las acciones, y el lenguaje de los hombres. En dos palabras: acciones humanas. Aunque espero que la expresión acciones humanas se entienda ya como pleonasmo, pues decir que algo es una acción ya implica que es un comportamiento humano. 
Retomando, una de las implicaciones epistemológicas de entender las acciones como objeto de estudio de las ciencias sociales, es que la noción de acción aclara un problema ya clásico de las investigaciones en ciencias sociales. Y es el problema de la posibilidad de conocimiento objetivo. ¿Es posible el conocimiento objetivo en ciencias sociales? Con base en lo que hemos anotado, podemos reformular el problema, y hacerlo más claro para el investigador de facto de las ciencias sociales, pues valga decir, que si bien el problema de objetividad en las investigaciones en ciencias sociales es de sobra comentado por los estudios de estas ciencias, la ambigüedad del mismo enturbia su clara comprensión. Así, si asumimos que las investigaciones en ciencias sociales son un conjunto de teorías verdaderas y justificadas de las acciones humanas, el problema de la objetividad podría reformularse diciendo, ¿es posible ofrecer teorías verdaderas y justificadas de las acciones humanas, sabiendo que las acciones humanas son causadas por estados públicamente no observables?

Aquí debemos retomar lo dicho anteriormente, en tanto las acciones humanas son causadas por razones (deseos y creencias) y éstas no son públicamente observadas, la pregunta se tornaría, entonces, en si es posible alcanzar objetividad en una serie de investigaciones en las que las causas de los objetos que se investigan no son públicamente observables.

En el caso de la migración, que es un caso representativo de las ciencias sociales, en algunos informes de migraciones, como en el trabajo de Mónica Isaza Bordamalo: Algunos imaginarios migratorios en Pereira y Dosquebradas (2009), puede verse la tensión por parte de los migrantes de manifestar las razones reales que los empujaron a viajar. Para la investigadora el asunto se hace aún más complejo cuando solicita a los entrevistados que describan el tipo de trabajo que realizan los migrantes. Isaza Bordamalo muestra cómo hay una incongruencia entre el número de emigrantes que dicen laborar formalmente, o en trabajos profesionales, y el porcentaje de emigrantes que no pagan impuestos $(64,5 \%)$, esto porque han de ocuparse en trabajos informales.

El punto es que para conocer las razones de la acción, la causa de las acciones, es necesario apelar a las creencias y deseos de las personas, pero estos, en tanto son estados mentales, no son públicamente observables ni cuantificables. De ahí la necesidad de la investigadora de apelar a fuentes como la introspección, o la interpretación para lanzar hipótesis, y ofrecer descripciones coherentes de su región de estudio.

A pesar de esto, valga la pena otra aclaración filosófica. En filosofía se entiende objetividad como un predicado de los juicios o proposiciones, así, hay juicios que son epistémicamente objetivos y juicios que no son objetivos, llamémoslos juicios epistémicamente subjetivos. Un juicio es epistémicamente objetivo cuando su valor de verdad es independiente de los intereses, deseos, y caprichos de su emisor. Un juicio es epistémicamente subjetivo cuando su valor de verdad depende de los caprichos e intereses de su emisor. Decir que: "existen básicamente dos factores que motivan la emigración de los habitantes de Pereira y Dosquebradas: el factor económico y el factor estudio", es un juicio epistémicamente objetivo, porque su valor de verdad no depende del capricho o interés de quien lo formuló; decir: "mi futuro no está en Colombia sino en España", es un juicio epistémicamente subjetivo, porque su valor de verdad depende de los intereses, deseos y caprichos de quien lo formuló.

Esto revela que es posible formular juicios epistémicamente objetivos en ciencias sociales. Cuando se revisa el trabajo de Mónica Isaza Bordamalo de la migración, y repito, éste es un caso que sirve como una muestra de investigación 
en ciencias sociales, puede verse que las afirmaciones en él consignadas son verdaderas independientemente de los caprichos, deseos e intereses del investigador. Afirmar descuidadamente que las investigaciones en ciencias sociales, por su naturaleza y diferencia con respecto a las ciencias naturales, están circunscritas a la subjetividad, implicaría que toda investigación en ciencias sociales es subjetiva, que los juicios que al respecto se formulen dependen de los prejuicios del investigador. Nadie cercano al fenómeno de la migración diría que cuando una investigación rigurosa y disciplinada arriba al juicio de que el factor económico es el factor predominante en la motivación de la población de emigrantes de Pereira y Dosquebradas su juicio está basado en su subjetividad.

\section{Ontología social, epistemología social}

Académicos de las ciencias sociales, que a nivel nacional han logrado abrir un horizonte de investigación en las disciplinas que abordan, coinciden en la necesidad de abastecer a la investigación en las distintas áreas de las ciencias sociales de modelos de pensamiento, que estudien desde la singularidad de los problemas sociales los fenómenos. Uno de los casos significativos en Colombia es el de Augusto Ángel Maya. En el artículo "Augusto Ángel Maya: aportes de Caldas al pensamiento y movimiento ambiental de Colombia", del profesor Isaías Tobasura Acuña, se describe la favorable influencia que en torno a las investigaciones ambientales ha tenido este doctor experto en pensamiento ambiental; sus improntas de trabajo han sido incluir de manera decisiva (i) los rasgos culturales y de poder que constituyen relevantemente en el objeto que se investiga, así como (ii) asumir modelos de investigación no homogenizantes. En el primer caso, se tiene un supuesto en la ontología social, es decir, una consideración con respecto a la naturaleza esencial de los objetos de investigación de los científicos sociales. En el segundo caso, un supuesto epistemológico, Ángel Maya prescribe la superación del monismo metodológico, poco exitoso para el reconocimiento de los rasgos singulares, rasgos que son condición necesaria en la investigación social.

Asumir que las ciencias sociales tienen por objeto de estudio las acciones humanas, y que éstas están causadas por creencias y por deseos, permite ver que cada uno de los problemas que los investigadores sociales enfrentan, implican necesariamente el reconocimiento de la singularidad de las comunidades humanas y su injerencia en la constitución de los objetos que se investigan; las maneras singulares como los humanos interactúan con su entorno (creencias), así como los intereses y apetencias que instauran en él (deseos), configuran la realidad social en la que anidan los objetos de investigación del científico social. Al respecto Dunn y Dobzhansky en el libro Herencia, raza y sociedad afirman: "De la aceptación de las diferencias individuales a la aceptación de las diferencias entre grupos sólo hay un paso; y la mayor parte de los pueblos, tanto primitivos como civilizados, tienen clara conciencia de sus diferencias con respecto a otros pueblos" (1975, p. 11).

En el caso del problema ambiental, ontología de estudio de las investigaciones ambientales, como lo manifiesta Tobasura Acuña en el artículo ya mencionado, son la adopción de ciertos estilos de vida el núcleo del problema ambiental. Así, las creencias de que el bienestar y la felicidad social están en minimizar el esfuerzo humano, y los deseos de satisfacer, a través de la tecnología, las apetencias de vivir una vida menos determinada por el esfuerzo físico, determinan la base causal del problema ambiental. En un ejemplo palmario Tobasura Acuña recrea el problema: 
La agradable facilidad de la vida moderna se está pagando cara. La tecnología ha ahorrado esfuerzo humano, pero en ocasiones puede resultar nefasta para el equilibrio de la vida e incluso para el bienestar del hombre. Los aerosoles evitan el pequeño esfuerzo de accionar una bomba manual para dispersar los insecticidas caseros, pero están debilitando la capa de ozono, ese escudo atmosférico que defiende la vida de los rayos ultravioletas. El cáncer de piel hoy es una amenaza real. (2009, p. 6)

El reconocimiento de que las comunidades culturales constituyen los hechos de estudio de las ciencias sociales (ontología social), dirige el foco de la investigación social hacia el estudio de las creencias y deseos que causan justamente estos hechos. Esto es dar un paso más allá de la propuesta de Emile Durkheim. En Las reglas del método sociológico, Durkheim ya había afirmado la existencia de una serie de hechos, que si bien no son físicos, son tan reales y objetivos como los hechos físicos u orgánicos. Los hechos sociales son para este sociólogo francés formas de obrar, de pensar y sentir que coaccionan los comportamientos de los individuos. Y si bien están soportados por las acciones sociales Durkheim afirma categóricamente que hay que tratarlos como cosas, no como ideas. Afirma:

Y sin embargo los fenómenos sociales son cosas y se les debe tratar como tales. Para demostrar esta proposición no es necesario filosofar sobre la naturaleza, ni discutir las analogías que presenten con los fenómenos de los reinos inferiores. Basta comprobar que son el único datum ofrecido al sociólogo. En efecto, se entiende por cosa todo lo que es dado, todo lo que se ofrece, o, más bien, todo lo que se impone a la observación. Tratar los fenómenos como cosas es tratarlos en calidad de data que constituye el punto de partida de la ciencia. Los fenómenos sociales presentan indiscutiblemente este carácter. (1999, p. 59)

No obstante, la observación de objetos, en este caso, de objetos que fungen como hechos sociales, no es suficiente para teorizar científicamente, teorización que deja de ser un asunto ontológico para volverse epistemológico. Como lo señala Karl Popper en su libro Conjeturas y refutaciones, el desarrollo del conocimiento científico, un hombre que se dedica a hacer descripciones de todas las cosas que ha observado durante su vida, poco aporta a la ciencia. Pues una teoría científica no es simplemente un catálogo bien ordenado de datos observacionales. La ciencia es explicativa, y por esto busca las causas de los fenómenos que observa. Si, siguiendo a Durkheim, los hechos sociales (ontología social) son los fenómenos que estudian las ciencias sociales, entonces las teorías de las ciencias sociales deben explicar este tipo de hechos, deben encontrar las causas que los producen. La literatura filosófica afirma que las creencias y deseos son las causas de los acontecimientos humanos y sociales. Es muy sensato afirmar que en el contexto de la investigación social, buscar las creencias y los deseos de los individuos y sociedades es muy promisorio para adelantar en la investigación en ciencia social.

\section{Razones externas y razones internas}

Quisiera finalizar mostrando cómo los conceptos que se involucran en la filosofía de la acción, pueden ser útiles para el investigador en ciencias sociales. Si bien inicialmente como herramienta heurística para la investigación.

Ya hemos establecido que las causas de las acciones humanas son las razones. Y que las razones son creencias más deseos. Así, las guerras, los partidos políticos, los presidentes, los torneos de fútbol, las migraciones humanas, los daños ambientales, las composiciones musicales, las novelas, todos en cuanto 
acciones humanas son provocados por creencias y deseos. Algo tan simple como la acción de mover mi mano para tomar agua, una acción básica, obedece a mi deseo de refrescar mi garganta, y a mis creencias de que lo que hay en el vaso es agua, y de que el agua sirve para alivianar la garganta después de haber hablado mucho. Nótese que una cosa es creer y otra es desear. Las creencias son verdaderas o falsas, los deseos son satisfechos o insatisfechos, nunca verdaderos o falsos. Si creo que lo que hay en el vaso es agua y efectivamente ese líquido se encuentra en el vaso, mi creencia es verdadera, en caso, de que lo que haya en el vaso sea vodka y no agua, mi creencia será falsa. En cambio, si deseo que la Selección de Brasil gane el Mundial de Fútbol, y la selección lo hace, mi deseo no fue verdadero, sino satisfecho, así como mi deseo no será falso, en caso de que la Selección de Brasil no lo gane.

En las razones para la acción, los deseos fungen como el fin al que se quiere llegar antes de realizada la acción. Las creencias son el conocimiento relevante que permite determinar cuáles son los medios para alcanzar el fin. Mi deseo de beber agua es el fin, y sé, en tanto mis creencias son verdaderas y están justificadas, que asiendo el vaso y llevándolo a la boca puedo satisfacer mi deseo, o también sé que si me dirijo a la cafetería y compro una botella de agua igualmente puedo satisfacer mi deseo. Dentro de las razones para la acción, las creencias son el componente cognitivo que configura a las acciones, y los deseos el componente motivacional. Hoy la afirmación de Hume de que la razón es y debe estar al servicio de las pasiones (1995), puede leerse que en tanto nuestras creencias se asumen como criterios para actuar, las creencias están subordinadas a nuestros deseos o motivaciones. Así, cuando nuestro conocimiento está dirigido a la acción, nuestro conocimiento se subordina a nuestros deseos.

A partir de la idea de que los deseos son los motivadores para actuar, y las creencias los medios para la acción, filósofos como Harry Frankfurt y John Searle clasifican las razones para actuar en dos: razones internas y razones externas. Tanto Searle como Frankfurt, el primero en su libro Razones para actuar (2000) y el segundo en su libro Necesidad, volición y amor (2007), muestran que los hombres no se conducen únicamente siguiendo sus deseos, sino también a partir de los deseos de otros. Así, argumentan a favor de la clasificación de razones internas y razones externas de las acciones. Grosso modo, la clasificación obedece al tipo de deseo que se persigue. Cuando el deseo obedece al querer individual, tenemos una razón interna, cuando obedece a un querer o deseo, que supera el querer del individuo, hablamos de razones externas. Para Searle los hechos institucionales, aquellos que dependen del acuerdo humano para su existencia, todos ellos son razones externas, dado que son hechos constituidos por creencias y deseos, en donde el querer del individuo se pierde por el querer del otro o de la colectividad. Y si bien, el querer del individuo puede coincidir con el querer del otro, en tanto el individuo excluya de su conciencia el querer individual lo hará por una razón externa, en tanto lo hagan considerando sus deseos propios lo harán por una razón interna.

Retomemos el estudio de migración citado anteriormente y la investigación de William Mejía Ochoa (2008) con respecto a las razones de la migración en el área de Pereira y su zona metropolitana, para ejemplificar el caso de las razones externas y las razones internas expuestas por Searle y Frankfurt. Más de la mitad de los emigrantes lo hacen por trabajo, alrededor de un $20 \%$ lo hacen por estudios, y el restante para reunirse con sus parientes o por violencia de grupos armados. Los migrantes que lo hacen por trabajo, forman un gran grupo de personas que viaja para poder enviarle dinero a sus familiares, y como los describe Isaza Bordamalo, los trabajos están distantes a los quereres de los individuos que los ejecutan; citando a Isaza (2009), trabajan cuidando niños y 
ancianos, en restaurantes o, en el mejor de los casos, en mecánicos y rutinarios empleos de fábricas. Qué podemos colegir de esto; que la mayoría de los colombianos emigran siguiendo razones externas, y en esta medida subordinan sus quereres individuales por los quereres de otros. $Y$, cetiris paribus, un muy bajo porcentaje emigra siguiendo razones internas.

Otro tanto, y más dramático puede decirse del lamentable fenómeno humano del desplazamiento forzado, el que es un tipo especial de migración. En el desplazamiento forzado un grupo de personas emigran de sus regiones por causas que afectan traumáticamente el conjunto de creencias y deseos que rutinariamente guían sus acciones. A diferencia de la migración de colombianos a otros países, en donde las razones de otros, casi siempre seres queridos, hijos, padres, hermanos promueven la partida (razones externas), en el desplazamiento forzado, los conflictos violentos y la violación a los derechos humanos provocan la aparición de este fenómeno. La Consultoría para los Derechos Humanos y el Desplazamiento (CODHES), en su boletín informativo de 2010 muestra que en Colombia el número de desplazados ya es cercano a los 5 millones de personas. En total 4. 915.579 millones de personas han sido víctimas del desplazamiento forzado en los últimos 25 años en Colombia. En términos de Durkheim, es un hecho social tan objetivo como los hechos físicos, el desplazamiento forzado en Colombia. No obstante, el observar el hecho es insuficiente para su compresión. Afirmar que la violencia y la violación a los derechos humanos es la causa del desplazamiento forzado, es apenas el inicio de una más comprensible explicación causal del fenómeno. La violencia y la violación de los derechos humanos, a pesar de ser hechos sociales, cosas en términos de Durkheim (1999), se instauran en el tipo de decisiones que promueven ciertas acciones de los violentos. Son las creencias y los deseos de los violentos las causas directas del desplazamiento forzado, y no la decisión y la acción de los violentados de migrar. Por esto, y como lo sugiere Searle, en tanto las acciones no sean causadas por el posible itinerario de deseos y creencias de las personas, éstas no son propiamente acciones que ellos ejercen, sino sucesos que padecen. En Razones para actuar Searle dice:

Supongamos que usted está leyendo esto sentado en una silla. A menos que haya algo radicalmente distinto en su situación -que usted esté, por ejemplo, atado a la silla, paralizado- usted también se da cuenta de que podría estar haciendo una gran cantidad de otras cosas. Podría estar leyendo algo distinto, podría llamar por teléfono a un viejo amigo, o salir a tomar una cerveza, para mencionar solo un puñado de posibilidades. Este sentido de las posibilidades de alternativas está incorporado en la estructura de las acciones humanas y nos da la convicción de que somos libres. (2000, p. 98)

Lo que implica que la libertad, entendida como la conciencia de la posibilidad de varias opciones de decisión y de acción, es condición necesaria para el establecimiento de las razones, ya sean internas o externas. En el caso del desplazamiento forzado, los que migran no poseen las razones de esta acción, pues ellos padecen la negación de opciones de decisión, y sin éstas no hay razones para la acción. Son los violentos los que deciden el rumbo de los miles de desplazados colombianos, y son ellos, antes que los campesinos que hoy intentan sobrevivir en las ciudades, el objeto de estudio de este fenómeno, el lugar donde reside su explicación.

Creencias, deseos, razones como causas, razones internas y razones externas, determinismo causal, actos de la volición son parte del marco conceptual que ofrece la filosofía de la acción para considerar sinópticamente los fenómenos humanos. Durkheim (1999) al asumirlos como objetos, dado que son exteriores y 
presentan coacciones a las personas, abrió el camino para la genuina investigación científica en ciencia social, pero al asumir el arsenal de conceptos que los recientes filósofos sociales y de la mente descubren en la explicación de las acciones humanas, se da un paso más en la comprensión de los fenómenos sociales, pues se puede instaurar el lugar propio de la explicación y producción de dichos hechos. El estudio filosófico de estos conceptos puede hacer más promisorio el tipo de investigación de los científicos sociales, aquél en donde se ha de buscar las razones para la acción. Fenómenos como la migración o la relación medio ambiente - hombre, en los que es apremiante un aguda comprensión desde renovados marcos conceptuales, como lo sugiere Augusto Ángel Maya, la filosofía de la acción puede revelarlos desde nuevas y razonadas perspectivas; todo esto, cuando entran a formar parte del itinerario de investigación de los científicos sociales los conceptos que explican las razones humanas para actuar.

\section{BIBLIOGRAFÍA}

- Aristóteles. (1967). Obras completas. Madrid: Aguilar.

- Consultoría para los Derechos Humanos y el Desplazamiento. (2010) ¿Salto estratégico o salto al vacío? Vía internet en: http://www.codhes.org/index.php?option=com_docman\&task=cat_vie w\&gid=62\&ltemid=50. (Consultado: 17 de marzo de 2010).

- Dunn, L.C.; Dobzhansky, T. (1975). Herencia, raza y sociedad. México: Fondo de Cultura Económica.

- Durkheim, Emile. (1999). Las reglas del método sociológico. Navarra: Folio.

- Forero, Édgar. (2003). El desplazamiento forzado en Colombia. Vía internet http://www.ideaspaz.org/eventos/download/edgar_forero.pdf (Consultado 15 de marzo de 2010).

- Frankfurt, Harry. (1975). Three concepts of free actions. En: Aristotelian Society. Vol. 49, pp. 95-125

- $\quad$ (2006). La importancia de lo que nos preocupa. Buenos Aires: Katz.

- $\quad$ (2007). Necesidad, volición y amor. Buenos Aires: Katz.

- Hollis, Martin. (1998). Filosofía de las ciencias sociales. Barcelona: Ariel.

- Hume, David. (1995). Investigación sobre el conocimiento humano. Barcelona: Altaya.

- Isaza, Mónica. (2009). Algunos imaginarios migratorios en Pereira y Dosquebradas. Tesis para optar el título en la maestría en Análisis Económicos e Internacionales Contemporáneos. Instituto de Altos Estudios para el Desarrollo, Bogotá.

- Mejía, William. (2008). Migraciones internacionales en Risaralda, un asunto bastante importante que requiere políticas subnacionales. Informe del Grupo de Investigación en Movilidad Humana, Red de Universidades del Eje Cafetero, Alma Mater.

- Popper, Karl. (1994). Conjeturas y refutaciones, el desarrollo del conocimiento científico. Barcelona: Paidós.

- Posada, Gregorio. (2007a). La noción tripartita del conocimiento, una introducción a la epistemología. Manizales: Universidad de Caldas.

- . (2007b). Una aproximación al problema de la acción. En: Jurídicas, Vol. 4, pp. 79 - 91

- Searle, John. (1979). what is an intentional State? En: Mind, New Series. Oxford. 
- (2000). Razones para actuar. Madrid: Círculo de Lectores.

- (2004). Libertad y neurobiología. Barcelona: Paidós.

- Tobasura, Acuña. (2009, Ene.-Jun.). Augusto Ángel Maya: aportes de Caldas al pensamiento y movimiento ambiental colombiano. En Luna Azul, No. $28 . \quad$ Vía internet: http://200.21.104.25/lunazul/downloads/Lunazul28_7.pdf. (Consultado 28 de marzo de 2010).

- Russell, Bertrand. (1950). Análisis del espíritu. Buenos Aires: Paidós.

- Von Wright, George. (1979). Explicación y comprensión. Madrid: Alianza editorial.

1. Las ideas que este artículo analiza forman parte de algunos de los temas de la línea de investigación en Filosofía de la Acción de la Universidad del Quindío. La tesis central fue expuesta en la Jornada Internacional sobre Migración Internacional y Retorno, en la Universidad Tecnológica de Pereira. Agradezco a la Red Alma Mater, y en especial a Ana María Murillo y William Mejía, quienes como organizadores del evento lograron reunir un destacado grupo interdisciplinar; las opiniones que en el encuentro se dieron han sido valiosas para la redacción final de este artículo.

2. Filósofos como Arthur Danto, Elizabeth Anscombe, John Austin, George Henry Von Wright, y recientemente, Donald Davidson, John Searle y Harry Frankfurt, analizan el comportamiento humano sobre la base de que las razones son la explicación causal del comportamiento humano. 